\title{
OsRLCK 57, OsRLCK107 and OsRLCK118 Positively Regulate Chitin- and PGN- Induced Immunity in Rice
}

\author{
Zhangqun $\mathrm{Li}^{\dagger}$, Ying $\mathrm{Ao}^{\dagger}$, Dongru Feng ${ }^{\dagger}$, Jun Liu, Jinfa Wang, Hong-Bin Wang and Bing Liu* (i)
}

\begin{abstract}
Background: The receptor-like cytoplasmic kinase, OsRLCK176 has been reported to participate in both chitin- and PGN-induced immunity in rice. Here, we further researched the function of the homologous proteins, OsRLCK57, OsRLCK107 and OsRLCK118, in chitin- and PGN immunity in rice.

Findings: Silencing of OsRLCK57,OsRLCK107 and OSRLCK118 suppressed chitin- and PGN-induced immunity responses, including reactive oxygen species generation, defense gene expression. Furthermore, OsRLCK107 could interact with OsCERK1 in a MAMP induced way, which suggested a possible physiological relevance of OsRLCKs107 to OsCERK1 pathway.
\end{abstract}

Conclusions: OsRLCK57, OsRLCK107 and OsRLCK118, positively regulate chitin- and PGN- induced responses in rice, similar to that observed in OsRLCK176.

Keywords: Receptor-Like Cytoplasmic Kinases, Microbe-Associated Molecular Pattern, Pattern Triggered Immunity, Rice

\section{Findings}

Plants are constantly challenged by a broad diversity of microbes in the environment. As sessile organisms without specialized immune cells, plants have evolved an elegantly sophisticated defense system, known as "innate immunity", to identify and eliminate the effects of detrimental invaders (Ausubel 2005). Microbe-associated molecular pattern (MAMP) triggered immunity (PTI) functions at the early stage of innate immunity (Schwessinger and Ronald 2012). The PTI response includes perceptions of MAMPs by pattern recognition receptors (PRRs) at the cell surface, followed by subsequent signal transduction into the cytoplasm to trigger immunity. At the cell surface, lysin motif (LysM) containing receptor-like kinases (RLKs), or non-kinase receptor proteins (RLPs), are the major receptors of oligosaccharidic MAMPs, such as chitin or peptidoglycan (PGN); examples are CEBiP for chitin, LYP4

\footnotetext{
* Correspondence: liubing@mail.sysu.edu.cn

${ }^{\dagger}$ Equal contributors

State Key Laboratory of Biocontrol and Collaborative Innovation Center of Genetics and Development, Guangdong Provincial Key Laboratory of Plant Resources, School of Life Sciences, Sun Yat-sen University, 510275 Guangzhou, People's Republic of China
}

and LYP6 for chitin and PGN in rice (Kaku et al. 2006; Liu et al. 2012), CERK1, LYK4 and LYK5 for chitin, and LYM1 and LYM3 for PGN in Arabidopsis (Cao et al. 2014; Miya et al. 2007; Wan et al. 2012; Willmann et al. 2011). After perception of MAMPs, a ligand-inducible complex can form to trigger downstream PTI responses. With regard to the signaling components immediately downstream of PRRs, receptor-like cytoplasmic kinases (RLCKs) have been suggested to play critical roles in delivering the immune responses (Macho and Zipfel 2014). Several RLCKs, especially those in subfamily VII, have been reported to participate in PTI in plants. In rice, examples are OsRLCK185 in chitininduced immunity and OsRLCK176 in both PGN- and chitin-induced immunity in rice (Ao et al. 2014; Yamaguchi et al. 2013). In Arabidopsis, besides the well-characterized roles of BIK1 and PBL1 in flg22-induced immunity, the ortholog of OsRLCK185, PBL27, also functions as a downstream component of CERK1 and contributes to chitin-induced immunity (Lu et al. 2010; Shinya et al. 2014). Recently, Zhou et al. reported that OsRLCK57, OsRLCK107, OsRLCK118 and OsRLCK176 are involved in XA21-mediated immunity and silencing of these genes also compromised the up-regulation of two 
PR genes after chitin treatment (Zhou et al., 2016). Since OsRLCK57, OsRLCK107, OsRLCK118 and OsRLCK176 all belong to RLCK group VIIa (Fig. 1a, Additional file 1: Figure S1) and we previously reported that OsRLCK176 is involved in the chitin and PGN signal response in rice (Ao et al. 2014), we were interested to determine the function of these homologous proteins, OsRLCK57, OsRLCK107 and OsRLCK118, in chitin and PGN immunity in this study.

Firstly, we identified the cell surface localization of OsRLCK57, OsRLCK107 and OsRLCK118 by the green fluorescent protein (GFP) fused OsRLCKs in

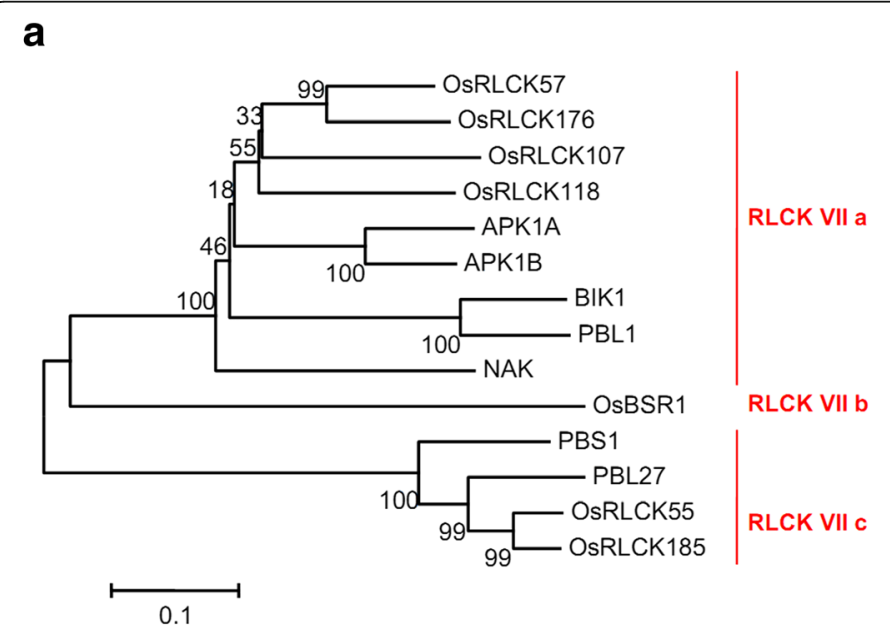

\section{C}

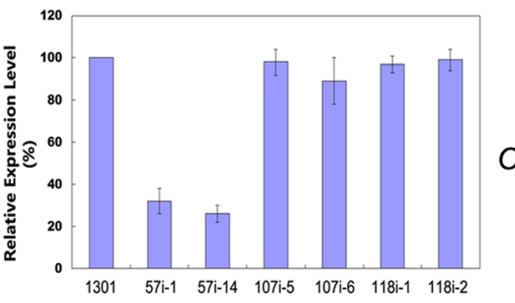

OsRKCK57

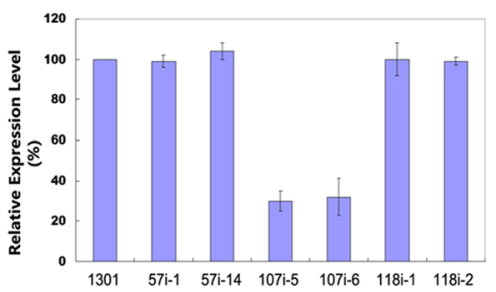

OsRLCK107

b

GFP
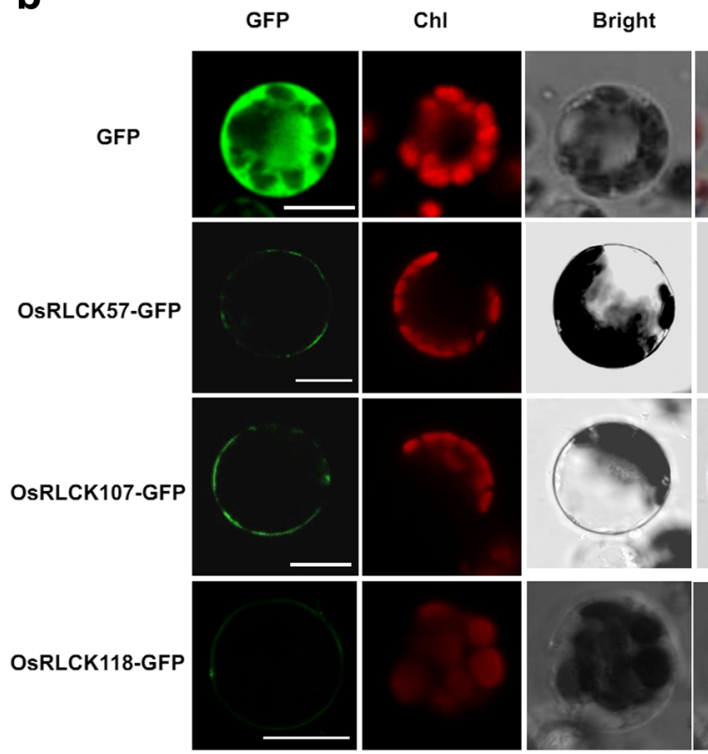

Merged
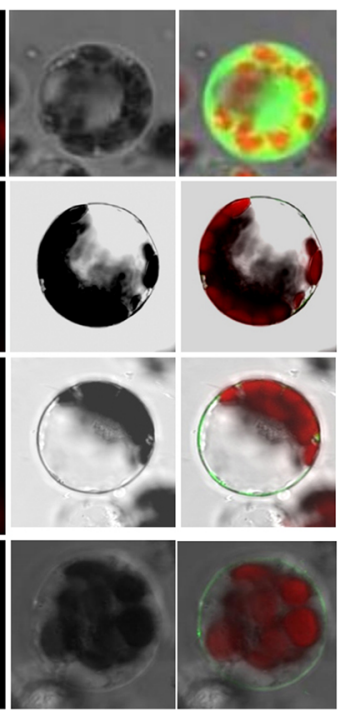

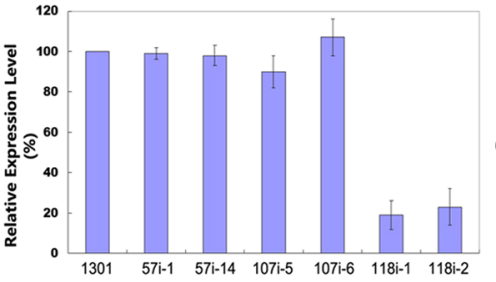

OsRLCK118

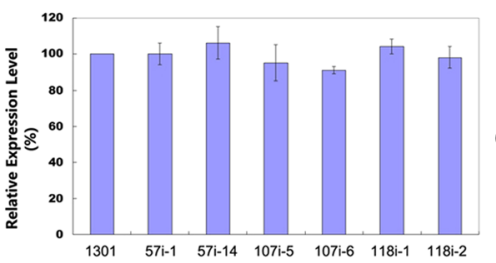

OsRLCK176

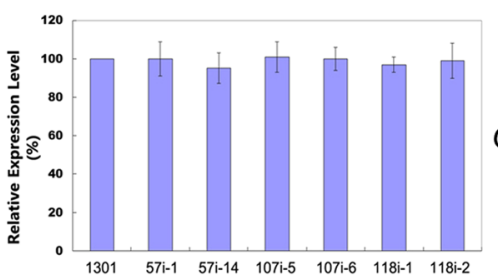

Fig. 1 Characterization of homologous OsRLCK57,OsRLCK107 and OsRLCK118. a OsRLCK57, OsRLCK107, OsRLCK118 is highly homologous with OsRLCK176 and BIK1. The phylogenetic tree was generated by MEGA4. Full length amino acid sequences were selected to generate the bootstrap neighbor-joining phylogenetic tree. Bootstrap probabilities were obtained from 1000 replicates. Scale bar is indicated. b OsRLCK57, OsRLCK107 and OsRLCK118 is plasma membrane localized protein. Subcellular localization of OsRLCK57, OsRLCK107 and OsRLCK118 was visualized using confocal microscope after transiently transformed OsRLCK57-GFP, OsRLCK107-GFP and OsRLCK118-GFP into rice protoplasts. Chl indicated the chlorophyll autofluorescence. Three biological replicates were performed, similar results were obtained. Bar $=10 \mu \mathrm{m}$. c Expression identification of RLCKs in different RNAi lines. The relative expression levels OsRLCK57, OsRLCK107 and OsRLCK118 were examined by quantitative RT-PCR in OsRLCK57, OsRLCK107 and OsRLCK118 RNAi lines, respectively. The expression of OsRLCK176 and OsRLCK185 was also examined in these RNAi lines to exclude its effects. Empty vector (1301) transgenic callus cells were used as control. 57i-1 and 57i-14: RNAi lines of OsRLCK57. 107i-5 and 107i-6: RNAi lines of OsRLCK107. 118i-1 and 118i-2: RNAi lines of OsRLCK118. The data represents the mean \pm standard deviation (SD) from three biological replicates 
rice protoplasts (Fig. 1b, Additional file 2: Material and Methods). Furthermore, the RNA interference (RNAi) lines with reduced expression of OsRLCK57, OsRLCK107 or OsRLCK118, respectively, but not other homologous OsRLCKs, were generated to probe the biological function of these OsRLCKS in chitin and PGN signal transduction (Fig. 1c, Additional file 2: Materials and Methods). Two representative RNAi lines were used for each gene in the subsequent analysis and the empty vector (1301) transformed callus was used as a control line. We investigated the reactive oxygen species (ROS) burst, one of the well-established early defense responses in plant immunity, after both chitin and PGN elicitation. We found that production of ROS was significantly induced by chitin in control line 1301, while ROS production was remarkably reduced in these OsRLCKs RNAi lines except the OsRLCK118 lines (Fig. 2a). The ROS production was reduced to $12-27 \%$ in the OsRLCK57 RNAi lines and to 14-17\% in the OsRLCK107 lines, respectively, compared with control line (Fig. 2a). In
PGN treatment assay, all of the ROS production was reduced in different OsRLCK RNAi lines, compared with control line 1301 (Fig. 2a). Inhibition of the ROS burst indicated that OsRLCK57, OsRLCK107 and OsRLCK118 could be involved in chitin- and/or PGN-induced immunity in rice, similar to OsRLCK176 and OsRLCK185 (Ao et al. 2014; Yamaguchi et al. 2013). Moreover, we investigated the activation of defense genes, a common indicator of the resistance response in plant immunity, in the RNAi lines. Four representative defense genes, OsPR5, OsPR10, PAL and PBZ1, which were previously examined in the OsRLCK176-RNAi lines and/or the OsRLCK185-RNAi lines, were assayed in this research (Ao et al. 2014; Yamaguchi et al. 2013). After chitin or PGN treatment, all of the four selected $P R$ genes were induced significantly in the control line, while expression of these genes was significantly suppressed in the OsRLCK-RNAi lines (Fig. 2b). As control, after another MAMP, LPS (lipopolysaccharide) treatment, the defense genes activation was only markedly

a

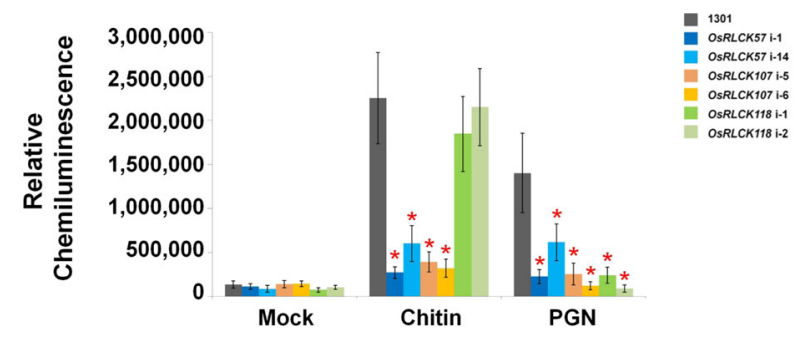

b
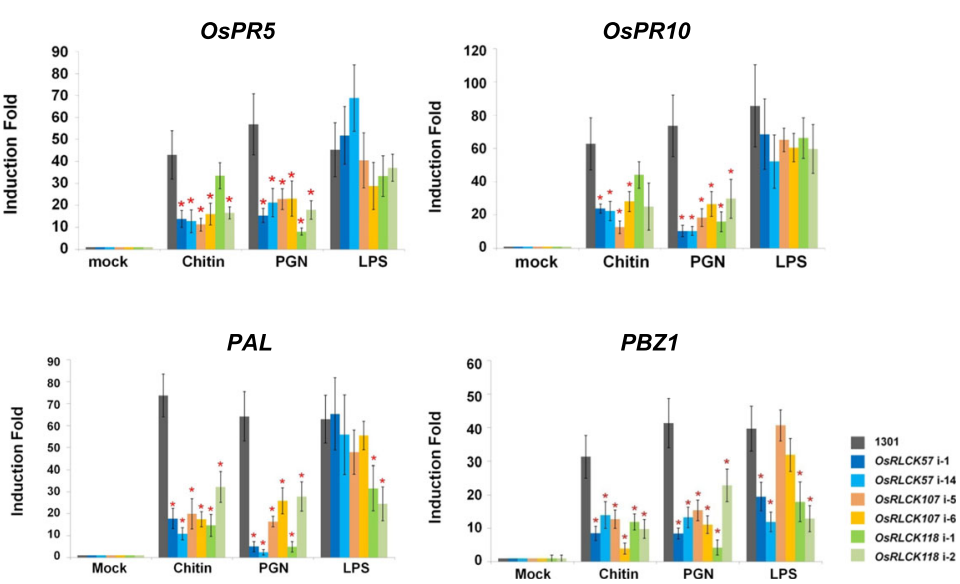

Fig. 2 OsRLCK57, OsRLCK107 and OsRLCK118 are involved in chitin- and PGN-triggered immunity in rice. a RNAi silencing of OsRLCKs compromise the burst of ROS induced by chitin and PGN. OsRLCK57-RNAi, OsRLCK107-RNAi, OsRLCK118-RNAi or empty vector (1301) transgenic callus cells were treated with $100 \mu \mathrm{g} / \mathrm{mL}$ chitin, PGN or sterile water (for mock) for 120 min in dark before assayed. The data represents the mean \pm standard deviation (SD) from three biological replicates. Statistically significant differences for OsRLCK-RNAi compared with the 1301 were calculated using Student's $t$-test and indicated by asterisks $\left({ }^{*} P<0.05\right)$. b RNAi silencing of OsRLCKs compromise the induction of PR genes by chitin and PGN. OsPR5, OsPR10, PAL and PBZ1 were determined by quantitative RT-PCR after $6 \mathrm{~h}$ treatments with $100 \mathrm{\mu g} / \mathrm{mL}$ chitin, PGN, LPS or sterile water (for mock) in dark. Empty vector (1301) transgenic callus cells were used as control. The data represents the mean \pm standard deviation (SD) from three biological replicates. Statistically significant differences for OsRLCK-RNAi compared with the 1301 were calculated using Student's $t$-test and indicated by asterisks $\left({ }^{*} P<0.05\right)$ 
suppressed in OsRLCK118-RNAi lines (for $P A L$ gene) and OsRLCK57 and OsRLCK118-RNAi lines (for PBZ1 gene) (Fig. 2b). Both the compromised burst of ROS and the activation of defense genes indicate that OsRLCK57, OsRLCK107, OsRLCK118 and OsRLCK107 play important roles in chitin- and PGN-induced defense responses in rice, just as OsRLCK185 and OsRLCK176 do.

In chitin- and PGN-trigged innate immunity in rice, OsCERK1 integrates extracellular chitin and PGN signals from different PRRs and delivers the immune signals to the cytoplasm by interacting with its cytoplasmic targets (Akamatsu et al. 2013; Ao et al. 2014; Shimizu et al. 2010; Yamaguchi et al. 2013). We were interested to determine whether OsRLCK57, OsRLCK107 and OsRLCK118 were correlated with OsCERK1. The Bimolecular fluorescence complementation (BiFC) assay indicated the interaction between OsRLCK107 with OsCERK1 (Fig. 3a). The interaction of OsRLCK107 with OsCERK1 was further confirmed by co-immunoprecipitation (coIP) analysis in rice protoplasts (Fig. 3b). However the interaction was not observed with OsRLCK55 and 118, possible due to the low expression of these proteins in our system. Interestingly, in the reported regulation mechanism of PRR-RLCKs in rice, OsRLCK176 and OsRLCK185 associate with OsCERK1 in the absence of MAMPs, and they then disassociate from OsCERK1 in the presence of MAMPs (Ao et al. 2014; Yamaguchi et al. 2013). Whereas the interaction between OsRLCK107 and OsCERK1 was enhanced in the presence of chitin or PGN (Fig. 3b), which distinguishes it from the reported actions of OsRLCK176 and OsRLCK185. The different mechanism of OsRLCKs in chitin and PGN signaling may indicate the complexity of the intracellular signaling network downstream of plasma membrane receptors.

PTI requires cytoplasmic members to link PRR activation with downstream signaling and RLCKs have emerged as key roles in the mechanism. In this research, we identified three members of RLCK group VIIa, OsRLCK57, OsRLCK107 and OsRLCK118, play roles in chitin and PGN immunity in rice. These results presented here will provide novel insight into the downstream signaling regulation of the chitin and PGN immune responses in rice.

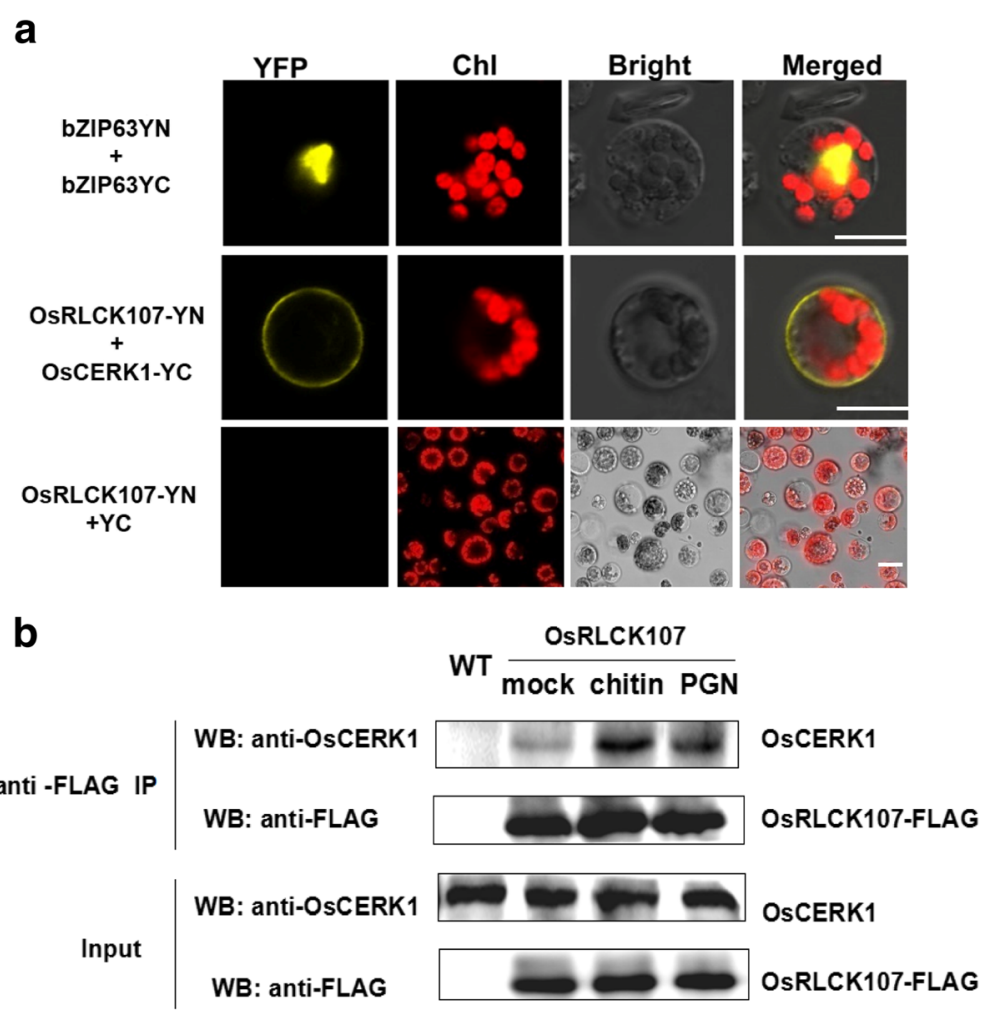

Fig. 3 OsRLCK107 interacts with OsCERK1. a OsRLCK107 interacts with OsCERK1 by BiFC. OsRLCKs-YN and OsCERK1-YC were co-expressed in rice protoplasts, the reconstituted YFP fluorescence was visualized using confocal microscope. Chl indicated the chlorophyll autofluorescence. Positive control: co-expression of bZIP63-YN and bZIP63-YC, negative control: co-expression of OsRLCKs-YN and blank-YC. Bar $=10 \mu \mathrm{m}$. b The interaction between OsRLCK107 with OsCERK1 is regulated by chitin and PGN. Protoplasts expressing OsRLCK107 with a C-terminal $3 \times$ FLAG epitope tag were treated with $150 \mu \mathrm{g} / \mathrm{mL}$ chitin、PGN or sterile water (for mock) for $10 \mathrm{~min}$. After treatment, protein was prepared for immunoprecipitation with anti-FLAG GEL (Sigma). The presence of OsCERK1 in the complex was analyzed by OsCERK1 antibody. Protein exacted from wide type (WT) protoplasts was used as negative control. Three biological replicates were performed and similar results were obtained 


\section{Additional Files}

Additional file 1: Figure S1. Aligments of full length amino acid sequences of OsRLCK57, OsRLCK107, OsRLCK118 and other RLCKs. Table S1. Primers used in this study. (DOC $6724 \mathrm{~kb}$ )

Additional file 2: Materials and Methods. (DOC $59 \mathrm{~kb})$

\section{Abbreviations}

MAMP: Microbe-Associated Molecular Pattern; PGN: Peptidoglycan; PRR: Pattern Recognition Receptor; PTI: Pattern Triggered Immunity; RLCK: Receptor-Like Cytoplasmic Kinase

\section{Acknowledgements}

This research was supported by the National Natural Science Foundation of China (No. 31100874 and No. 31425003), the Natural Science Foundation of Guangdong Province, PR China (No.S2011040001868), the Fundamental Research Funds for the Central Universities (12lgpy33 and 14lgzd04), and the project of Science and Technology New Star in Zhujiang City (2012-78).

\section{Authors' Contributions}

ZL, YA and DF performed research. JL analyzed data. BL, HBW and JW designed the research. All authors read and approved the final manuscript.

\section{Competing Interests}

The authors declare that no competing interests exist.

\section{Accession Numbers}

Gene sequence data referred to in this article can be found in the GenBank/ EMBL databases under the following accession numbers:

OsRLCK107(Os03g0274800), OsRLCK57(Os01g0973500),

OsRLCK118(Os03g0821900), OsRLCK176(Os05g0110900),

OsRLCK185(Os05g0372100), OsCERK1(Os08g0538300), OsPR5(Os01g0122000), OsPR10 (Os03g0300400), PAL (Os02g0627100), PBZ1 (Os12g0555500). [BIK1: AT2G39660, NAK: AT5G02290, PBL1: AT3G55450, PBL27: At5G18610, APK1A: AT1G07570, APK1B: AT2G28930, PBS1: AT5G13160, NAK: AT5G02290, PBL1: AT3G55450].

Received: 17 August 2016 Accepted: 14 February 2017

Published online: 21 February 2017

\section{References}

Akamatsu A, Wong HL, Fujiwara M, Okuda J, Nishide K, Uno K, Imai K, Umemura K, Kawasaki T, Kawano Y, Shimamoto K (2013) An OsCEBiP/OsCERK1OsRacGEF1-OsRac1 Module is an Essential Early Component of ChitinInduced Rice Immunity. Cell Host Microbe 13:465-476

Ao Y, Li Z, Feng D, Xiong F, Liu J, Li JF, Wang M, Wang J, Liu B, Wang HB (2014) OsCERK1 and OsRLCK176 Play Important Roles in Peptidoglycan and Chitin Signaling in Rice Innate Immunity. Plant J 80:1072-1084

Ausubel FM (2005) Are Innate Immune Signaling Pathways in Plants and Animals Conserved? Nat Immunol 6:973-979

Cao Y, Liang Y, Tanaka K, Nguyen CT, Jedrzejczak RP, Joachimiak A, Stacey G (2014) The kinase LYK5 is a major chitin receptor in Arabidopsis and forms a chitin-induced complex with related kinase CERK1. eLife 3:e03766

Kaku H, Nishizawa Y, Ishii-Minami N, Akimoto-Tomiyama C, Dohmae N, Takio K, Minami E, Shibuya N (2006) Plant Cells Recognize Chitin Fragments for Defense Signaling Through A Plasma Membrane Receptor. Proc Natl Acad Sci U S A 103:11086-11091

Liu B, Li JF, Ao Y, Qu J, Li Z, Su J, Zhang Y, Liu J, Feng D, Qi K, He Y, wang J, Wang HB (2012) Lysin Motif-Containing Proteins LYP4 and LYP6 Play Dual Roles in Peptidoglycan and Chitin Perception in Rice Innate Immunity. Plant Cell 24:3406-3419

Lu D, Wu S, Gao X, Zhang Y, Shan L, He P (2010) A Receptor-Like Cytoplasmic Kinase, BIK1, Associates With a Flagellin Receptor Complex to Initiate Plant Innate Immunity. Proc Natl Acad Sci U S A 107:496-501

Macho AP, Zipfel C (2014) Plant PRRs and the Activation of Innate Immune Signaling. Mol Cell 54:263-272

Miya A, Albert P, Shinya T, Desaki Y, Ichimura K, Shirasu K, Narusaka Y, Kawakami N, Kaku H, Shibuya N (2007) CERK1, a LysM Receptor Kinase, is Essential for Chitin Elicitor Signaling in Arabidopsis. Proc Natl Acad Sci U S A 104:19613-19618
Schwessinger B, Ronald PC (2012) Plant Innate Immunity: Perception of Conserved Microbial Signatures. Ann Rev Plant Biol 63:451-482

Shimizu T, Nakano T, Takamizawa D, Desaki Y, Ishii-Minami N, Nishizawa Y, Minami E, Okada K, Yamane H, Kaku H, Shibuya N (2010) Two LysM Receptor Molecules, CEBiP and OsCERK1, Cooperatively Regulate Chitin Elicitor Signaling in Rice. Plant J 64:204-214

Shinya T, Yamaguchi K, Desaki Y, Yamada K, Narisawa T, Kobayashi Y, Maeda K, Suzuki M, Tanimoto T, Takeda J, Nakashima M, Funama R, Narusaka M, Narusaka Y, Kaku H, Kawasaki T, Shibuya N (2014) Selective Regulation of the Chitin-Induced Defense Response by the Arabidopsis Receptor-Like Cytoplasmic Kinase PBL27. Plant J 79:56-66

Wan J, Tanaka K, Zhang XC, Son GH, Brechenmacher L, Nguyen TH, Stacey G (2012) LYK4, a Lysin Motif Receptor-Like Kinase, is Important for Chitin Signaling and Plant Innate Immunity in Arabidopsis. Plant Physiol 160:396-406

Willmann R, Lajunen HM, Erbs G, Newman MA, Kolb D, Tsuda K, Katagiri F, Fliegmann J, Bono J, Cullimore JV, Jehle AK, Götz F, Kulik A, Molinaro A, Lipka V, Gust AA, Nürnberger T (2011) Arabidopsis Lysin-Motif Proteins LYM1 LYM3 CERK1 Mediate Bacterial Peptidoglycan Sensing and Immunity to Bacterial Infection. Proc Natl Acad Sci U S A 108:19824-19829

Yamaguchi K, Yamada K, Ishikawa K, Yoshimura S, Hayashi N, Uchihashi K, Ishihama N, Kishi-Kaboshi M, Takahashi A, Tsuge S, Ochiai H, Tada Y, Shimamoto K, Yoshioka H, Kawasaki T (2013) A Receptor-Like Cytoplasmic Kinase Targeted by a Plant Pathogen Effector is Directly Phosphorylated by the Chitin Receptor and Mediates Rice Immunity. Cell Host Microbe 13:347-357

Zhou X, Wang J, Peng C, Zhu X, Yin J, Li W, He M, Wang J, Chern M, Yuan C, Wu W, Ma W, Qin P, Ma B, Wu X, Li S, Ronald P, Chen X (2016) Four ReceptorLike Cytoplasmic Kinases Regulate Development and Immunity in Rice. Plant Cell Environ 39:1381-1392

\section{Submit your manuscript to a SpringerOpen ${ }^{\circ}$ journal and benefit from:}

- Convenient online submission

- Rigorous peer review

- Immediate publication on acceptance

Open access: articles freely available online

- High visibility within the field

- Retaining the copyright to your article

Submit your next manuscript at $>$ springeropen.com 